\title{
U Scorpii 2010 outburst: observational evidence of an underlying ONeMg white dwarf ${ }^{\star}$
}

\author{
E. Mason \\ Space Telescope Science Institute (STScI), 3700 S. Martin Dr., Baltimore, MD 21218, USA \\ e-mail: emason@stsci.edu \\ Received 8 June 2011 / Accepted 18 July 2011 \\ ABSTRACT

\begin{abstract}
This paper presents U Sco nebular spectra collected in the period March-May 2010 after the binary outburst on Jan. 28, 2010. The spectra display strong $[\mathrm{Nev}]$ and [NeIII] lines that can be used to compute the relative abundance of [Ne/O]. The value obtained $([\mathrm{Ne} / \mathrm{O}]=1.69)$ is higher than the typical $[\mathrm{Ne} / \mathrm{O}]$ abundance found in classical novae from $\mathrm{CO}$ progenitors and suggests that $\mathrm{U}$ Sco has a ONeMg white-dwarf progenitor. It follows that U Sco will not explode as a SN Ia but rather collapse to become a neutron star or a millisecond pulsar.
\end{abstract}

Key words. novae, cataclysmic variables - stars: individual: USco

\section{Introduction}

The outburst of U Scorpii (USco) on Jan. 28, 2010 at $V_{\max }=$ $8.05 \mathrm{mag}$ (Munari et al. 2010) is the tenth outburst recorded for this nova and, thanks to the Schaefer monitoring and alert (see e.g. Schaefer et al. 2010), the best-studied of this kind together with the outburst of 1999. The importance of observing U Sco and recurrent novae ( $\mathrm{RNe}$ ) outbursts in general resides in the opportunity to understand the kinematics and the composition of RNe ejecta in relation to both classical novae (CNe) and supernovae (SNe). While observations (Della Valle \& Livio 1996) show that RNe are not the main contributors to the class of SN Ia progenitors, numerous theoretical works consider RNe within the single degenerate scenario as valid progenitors of type Ia $\mathrm{SNe}$ (e.g. Hachisu et al. 2000; Hachisu \& Kato 2002; Livio 2000, and reference therein; Nomoto et al. 2000; see also Podsiadlowski 2008). To better understand this problem, I collected X-Shooter multi-wavelength medium resolution spectra of U Sco during its late decline from the plateau phase down to quiescence. The goal was to characterize the physical parameters of the ejecta when they are optically thin and compute abundances, which are a discriminating factor between eroded white dwarfs (WDs) vs. noneroded WDs. In this Letter, I present nebular spectra of U Sco taken 45, 73, and 104 days after the outburst and discuss the measured $[\mathrm{Ne} / \mathrm{O}]$ abundance value in the attempt to identify the underlying WD. A more detailed discussion of the U Sco 2010 outburst spectral evolution will be presented in a separate paper (Mason et al., in prep.), which collects a larger sample of spectra.

\section{Observation and data reduction}

$\mathrm{X}$-Shooter is the first of the second-generation instruments installed on the VLT (D'Odorico et al. 2006). It is a three-arm spectrograph that allows medium-resolution spectroscopy in the wavelength range $300-2500 \mathrm{~nm}$ in a single exposure. The observations were performed in service mode within ideal time windows for each epoch. The spectra were taken on March 15

\footnotetext{
^ Based on observations carried out at the ESO's VLT+X-Shooter under program 284.D-5041.
}

(i.e. +46 days from maximum, at orbital phase 0.07), April 11 (+73 days, at orbital phase 0.01), and May 122010 (+104 days, at orbital phase 0.16). The instrument setup on March 15 was slit $0.8^{\prime \prime}, 0.7^{\prime \prime}$ and $0.6^{\prime \prime}$ for the UVB, VIS, and NIR arms, respectively; it was slit $1.0^{\prime \prime}, 0.9^{\prime \prime}$ and $0.6^{\prime \prime}$ for the April and May observations. The CCD readout was always $100 \mathrm{kHz}$ (high gain), but $1 \times 2$ binning was adopted for the April and May observations. The observing strategy involved nodding along the slit with a nod-throw of $5^{\prime \prime}$. The slit was always oriented along the parallactic angle at the beginning of each exposure and the selected telluric standard star (also observed nodding along the slit) was the Hipparcos star Hip 82254, a B3 v star of magnitudes $B=6.76 \mathrm{~V}=6.81$ and $H=6.92$. The data were reduced using the instrument pipeline, version 1.2.2, in "physical model mode" (Modigliani et al. 2010, see also Bristow et al. 2010) for image pre-processing, sky-subtraction and spectral rectification. Order extraction and merging, telluric correction, extinction correction, and flux calibration were performed with IRAF. The spectrophotometric standard EG 274, observed on May 12, was used to calibrate all spectra (see Vernet et al. 2008, for a UV to NIR database of spectrophotometric standard stars). The flux-calibrated spectra were aligned to the VIS arm and scaled to U Sco $J$ magnitude at the corresponding epoch (Schaefer, priv. comm.). The spectra were then dereddened adopting $E(B-V)=0.15$ (from the equivalent width of the $\mathrm{Na} \mathrm{D}_{1}$ interstellar absorption, Munari \& Zwitter 1997) and the upper and lower limits of 0.25 and $0.00 \mathrm{mag}$, respectively, which account for the large uncertainties resulting from the combination of different methods (e.g. Diaz et al. 2010).

\section{The nebular phase and the forbidden emission lines}

The sequence of collected spectra (Fig. 1) covers the U Sco outburst light curve from the second plateau after the super soft phase has ended ( $V \geq 16.5 \mathrm{mag}$ ) to the quiescent phase $(V \geq$ $18 \mathrm{mag}$, see, for example, Munari et al. 2010; Diaz et al. 2010, for the U Sco 2010 outburst light curve). The spectra show that beginning Mar. 15, U Sco entered the nebular phase, displaying 


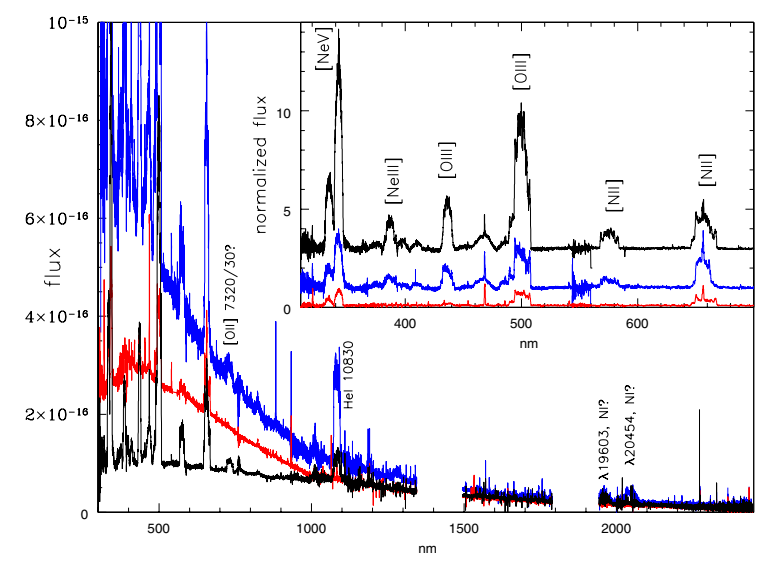

Fig. 1. U Sco X-Shooter spectra: Mar. 15 in blue, Apr. 11 in black and May 12 in red. In the inset: a portion of the same spectra (UVB+VIS) showing the nebular forbidden transitions. The spectra in the inset have been normalized to unity and offset vertically for clarity. They are sorted in order of their nebular line intensity for clarity. The displayed spectra have been median-filtered.

strong and broad emission lines of [NII], [OIII], [NeIII] and [Nev]. The nebular phase of U Sco was reported already by Diaz et al. (2010), who observed [NII], [OIII], and [NeIII] starting from day +75 after maximum. Though it has been claimed that U Sco does not develop forbidden transitions (e.g. Warner 1995) and that the 2010 outburst was the first time that the nova displayed a nebular spectrum (e.g. Diaz et al. 2010), neither statement is accurate. First, because the observations performed during the past outbursts were not sufficiently extended in time after the maximum; second, because the average spectrum of Thoroughgood et al. (2001, their Fig. 1) clearly shows a broad composite emission, which should be identified with [OIII] $\lambda 5007$. Its profile is very similar to that reported in this paper (see Fig. 1 inset).

The forbidden transitions appear when the U Sco continuum has significantly dimmed and the nova is about 3 mag fainter than during the first plateau phase. At this epoch the continuum varies not only with the time since maximum, but also with the orbital phase (see Fig. 1). The April spectrum was taken close to the eclipse time (at orbital phase 0.01) and shows a flat continuum. The March and May spectra (centered at orbital phase 0.07 and 0.16 , respectively) are characterized by a blue continuum. This can be explained by the hot blue component (the white dwarf itself or an accretion hot-spot) being masked by the secondary star at the time of the April observation.

The evolution of the broad emission lines from the ejecta is independent of the orbital phase, but their intensity is maximum when the continuum strength decreases. The three nebular spectra also show that the resonant transitions constantly weaken in time relative to the forbidden transitions, until they almost completely disappear by May 12 . This is indicative of a progressively lower density in the ejecta, though this is always relatively high when compared to the densities in planetary nebulae. The analysis of the line profiles shows that the $\mathrm{H} \alpha$ emission dominates the "6563 blend" in the March and April spectra, and that it is significantly weaker than the $\left[\mathrm{NII}_{\mathrm{II}}\right] 26584$ emission only in the May spectrum. Hence, only in the latter spectrum it is possible to use the $[\mathrm{NII}]$ lines ratio to constrain the ejecta density and temperature. At this epoch the $\left[\mathrm{N}_{\mathrm{II}}\right] \lambda(6584+6548) / \lambda 5755$ flux ratio is $\sim 6$, implying that collisions are contributing to the line formation and that the gas densities are higher than $10^{5} \mathrm{~cm}^{-3}$ (see e.g. Osterbrock \& Ferland 2006). High gas densities are suggested also by the [OIII] $\lambda(5007+4959) \lambda 4363$ flux

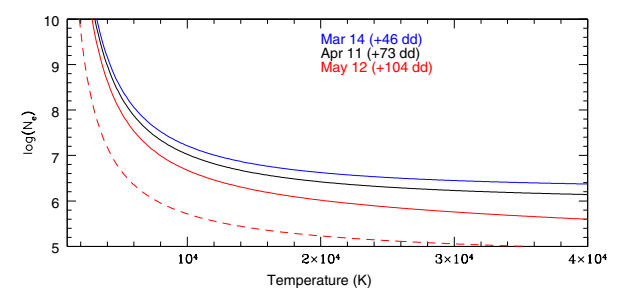

Fig. 2. Diagnostic diagram for the flux ratios $\left(F_{4959}+F_{5007}\right) / F_{4363}$ (solid lines) and $\left(F_{6548}+F_{6583}\right) / F_{5755}$ (dashed lines) at the three epochs Mar. 15 (blue), April 11 (black), and May 12 (red). Each line represents the loci of density and temperature that match the observed flux ratios. Fluxes correspond to the dereddened spectra.

ratio $^{1}$, which is 2.97, 4.79 and 9.37 in the March, April and May spectra, respectively, and indicates densities $\geq 5 \times 10^{6}-10^{7} \mathrm{~cm}^{-3}$ for temperatures in the nominal range $14000-10000 \mathrm{~K}$. Figure 2 plots the diagnostic diagram for the [OIII] and [NII] flux ratios. Note that the lower temperatures and densities indicated by the $[\mathrm{NII}] \lambda \lambda 6584,6548 /\left[\mathrm{N}_{\mathrm{II}}\right] \lambda 5755$ flux ratio are consistent with the fact that the $[\mathrm{NII}]$ transitions typically form in the outer and cooler shell of the expanding ejecta (e.g. Osterbrock \& Ferland 2006).

Though accurate elemental abundance determination requires the combined modeling of UV and optical observations (e.g., Schwarz 2002), a first oder approximation of the relative abundances can be computed by using the flux ratios of emission lines from ions with similar ionization potential energies, similar critical densities, and the same excitation mechanism (Kingdon \& Williams 1997). This method has the advantage of being fairly insensitive to temperature uncertainties (temperatures that differ by a factor of 2 imply uncertainties of $\sim 20 \%$ in the abundance) and has been tested against models for a range of temperatures and densities. In the case of the U Sco nebular spectra, it is possible to compute the $[\mathrm{Ne} / \mathrm{O}]$ abundance from the flux ratio of the lines [NeIII] 33869 and [OIII] 55007 , in both the March and April spectra. In the high-density limit (e.g. Dopita 2001) the line flux can be written as

$F_{i j}=N_{i} E_{i j} A_{j i} \frac{g_{j}}{g_{i}} \exp \left[\frac{-E_{i j}}{k T}\right]$,

where $i$ and $j$ are the two levels of the transition, $N_{i}$ is the density of level $i, E_{i j}$ is the energy of the transition, $A_{i j}$ is the transition probability, $g_{i}$ and $g_{j}$ are the statistical weights of the states, $k$ is the Boltzmann constant, and $T$ the gas temperature. Adopting $T_{\mathrm{e}} \sim 12000 \mathrm{~K}$, as derived from the above considerations and Fig. 2, one obtains the relative abundances of $[\mathrm{Ne} / \mathrm{O}]=1.97$ (March) and $[\mathrm{Ne} / \mathrm{O}]=1.69$ (April), as reported in Table 1. Kingdom \& Williams (1997) established that the $[\mathrm{NeIII}] \lambda 3869 /[\mathrm{OIII}] \lambda 5007$ pair is a good indicator of the $[\mathrm{Ne} / \mathrm{O}]$ abundance for gas densities $<10^{6} \mathrm{~cm}^{-3}$, while U Sco ejecta have densities that are about one order of magnitude higher. To quantify the uncertainty associated to the method in this case, I applied it to V382 Vel (Della Valle et al. 2002, their Table 3), a very fast nova, which developed strong [NeIII] optical emission lines in a high-density ejecta $\left(\simeq 10^{7} \mathrm{~cm}^{-3}\right)$, similarly to

${ }^{1}$ In computing these ratios, the conservative $\mathrm{H} \gamma$ flux $F_{\mathrm{H} \gamma}=0.85 \times F_{\mathrm{H} \beta}$ has been subtracted from the [OIII] $\lambda 4363$ emission, though the $\lambda 4363$ profile does not show strong evidence of a blend. The $\mathrm{H} \gamma / \mathrm{H} \beta$ ratio has been inferred from flat Balmer decrement $\mathrm{H} \delta / \mathrm{H} \beta \geq 0.7$ measured in both the March and April spectra. The derived flux ratios should, therefore, be taken as an upper limit. No $\mathrm{H} \gamma$ fractional contribution to the 4363 line has been assumed in the May spectrum, because, at that time, no broad emission component from the ejecta is detectable in any of the Balmer lines (and in $\mathrm{H} \delta$, in particular). 
Table 1. Integrated emission line fluxes of the ejecta as measured in the $\mathrm{X}$-Shooter dereddened spectra and, within brackets, the fluxes measured on the spectra before correction for reddening.

\begin{tabular}{lccc}
\hline \hline Line ID \& $\lambda(\AA)$ & \multicolumn{2}{c}{ Flux $\left(\times 10^{-15} \mathrm{erg} \mathrm{cm}^{-2} \mathrm{~s}^{-1} \AA^{-1}\right)^{\dagger}$} \\
& March 15 & April 11 & May 12 \\
\hline$[\mathrm{Nev}](1) 3343$ & $42.5(20.8)$ & $21.2(10.3)$ & $5.8(2.8)$ \\
{$[\mathrm{Nev}](1) 3426$} & $119.0(60.2)$ & $56.0(27.9)$ & $15.4(7.5)$ \\
{$[\mathrm{Ne}$ III] $](1) 3869$} & $41.2(18.7)$ & $12.4(6.1)$ & - \\
{$[\mathrm{NeIII}](1) 3967$} & $13.8(5.7)$ & $3.7(1.8)$ & - \\
$\mathrm{H} \delta$ & $12.0(5.6)$ & $2.7(1.1)$ & - \\
{$[\mathrm{OIII}](2) 4363$} & $60.2(33.2)$ & $19.4(11.4)$ & $2.6(1.4)$ \\
{$[\mathrm{OIII}](1) 5007+4956$} & $151.0(84.0)$ & $81.4(49.3)$ & $22.8(14.1)$ \\
{$[\mathrm{OIII}](1) 5007^{\dagger \dagger}$} & $102.0(63.0)$ & $59.8(37.0)$ & $16.9(10.5)$ \\
$\mathrm{H} \beta 4861$ & $17.0(11.3)$ & $3.5(2.5)$ & $0.6(0.4)$ \\
{$[\mathrm{NII}](3) 5755$} & $18.2(12.2)$ & $9.8(6.7)$ & $2.0(1.6)$ \\
$\mathrm{H} \alpha+[\mathrm{NII}](1)$ & $58.3(41.9)$ & $22.2(15.8)$ & $12.0(8.7)$ \\
\hline$[\mathrm{Ne} / \mathrm{O}]^{\dagger \dagger \dagger}$ & $1.97_{-0.19}^{+0.03}$ & $1.69_{-0.12}^{+0.02}$ & - \\
\hline
\end{tabular}

Notes. $\left.{ }^{(}\right)$Flux errors are in the range $1-30 \%$, depending on the line strength. ${ }^{(\dagger \dagger)}$ The $[\mathrm{OIII}] \lambda 5007$ flux has been computed assuming the theoretical transition probability of 3 after subtraction of the $\mathrm{H} \beta$ contribution. ${ }^{(\dagger \dagger)}$ The lower and upper limit in the $[\mathrm{Ne} / \mathrm{O}]$ abundances have been computed measuring the spectra corrected for reddening assuming $E(B-V)=0.25 \mathrm{mag}$ and the uncorrected spectra, respectively, also adding the appropriate uncertainty on the measured line flux.

U Sco. Comparing V382 Vel [Ne/O] abundance derived from the $\lambda 3869 / \lambda 5007$ flux ratio, with that obtained via photo-ionization modeling (Shore et al. 2003), one obtains relative errors in the range $25-50 \%$, depending on the epoch. Hence, the U Sco $[\mathrm{Ne} / \mathrm{O}]$ abundances could be as low as $0.99_{-0.10}^{+0.01}$ and $0.85_{-0.06}^{+0.01}$ for the March and April spectra, respectively. This would not change the conclusion derived in the next section.

\section{Discussion and conclusion: the ultimate U Sco fate}

The general consensus about SN Ia progenitors is that they originate from $\mathrm{CO}$ WDs accreting matter up to the Chandrasekhar limit either via stellar merging (double degenerate scenario where two CO WDs coalesce) or via mass transfer from a less evolved companion (single degenerate scenario). Within the single degenerate scenario the accreting WD ought to be massive (i.e. close to the Chandrasekhar limit), and accreting at a high rate $\left(\geq 10^{-8}-10^{-7} M_{\odot} \mathrm{yr}^{-1}\right.$, Nomoto et al. 2007, and reference therein, see also Yaron et al. 2005). Hence, RNe whose frequent outbursts are explained by massive WDs and high mass-transfer rates (e.g. Truran et al. 1988) remain viable candidates, contrary to what is suggested by the population synthesis simulations (Di Stefano 2010 and reference therein) and their census (Della Valle \& Livio 1996). U Sco, having a WD >1.37 $M_{\odot}$ (Hachisu et al. 2000; Thoroughgood et al. 2001) and accreting at a rate $>10^{-7} M_{\odot} \mathrm{yr}^{-1}$ (Hachisu et al. 2000; Matsumoto et al. 2003), seems very likely to explode as a SN Ia. However, it is also general consensus that an accreting ONeMg WD cannot lead to a SN Ia explosion but, eventually, to a core collapse and the formation of a neutron star or a millisecond pulsar (e.g. Nomoto $\&$ Kondo 1991). The high U Sco [Ne/O] abundance (when compared to the solar values of -0.76 ) is intriguing both because it points to dredged-up material and a possibly eroded WD, and because it provides information about the composition/nature of the underlying WD. Dredged-up material is typically associated with an eroded WD because, according to thermo-nuclear (TNR) computations (e.g. Prialnik \& Livio 1995), anything that is mixed within the H-layer where the TNR ignites is ejected
Table 2. Ne abundances for a sample of classical novae.

\begin{tabular}{lccc}
\hline \hline Nova & WD & $[\mathrm{Ne} / \mathrm{O}]]^{\dagger}$ & Ref. \\
\hline V693 CrA & ONeMg & +1.21 & Vanlandingham et al. (1999) \\
V1370 Aql & ONeMg & +1.76 & Livio \& Truran (1994) \\
V1974 Cyg & ONeMg & +0.47 & Vanlandingham et al. (2005) \\
V382 Vel & ONeMg & +0.66 & Shore et al. (2003) \\
V4160 Sgr & ONeMg & +0.69 & Schwarz et al. (2007a) \\
V838 Her & ONeMg & +1.61 & Schwarz et al. (2007a) \\
QU Vul & ONeMg & +0.92 & Schwarz (2002) \\
V1065 Cen & ONeMg & +0.74 & Helton et al. (2010) \\
LMC 1990 N.1 & ONeMg & +0.45 & Vanlandingham et al. (1999) \\
U Sco & ONeMg & +1.69 & this paper \\
RR Pic & $?$ & +0.94 & Livio \& Truran (1994) \\
V977 Sco & $?$ & +0.60 & Livio \& Truran (1994) \\
V1186 Sco & CO & -0.42 & Schwarz et al. (2007b) \\
LMC 1991 & CO & -1.65 & Schwarz et al. (2001) \\
QV Vul & CO & -0.96 & Livio \& Truran (1994) \\
HR Del & CO & -0.53 & Livio \& Truran (1994) \\
V1500 Cyg & CO & -0.069 & Livio \& Truran (1994) \\
V1688 Cyg & CO & -0.62 & Livio \& Truran (1994) \\
GQ Mus & CO & -1.09 & Livio \& Truran (1994) \\
PW Vul & CO & -0.67 & Livio \& Truran (1994) \\
V842 Cen & CO & -0.86 & Livio \& Truran (1994) \\
V827 Her & CO & -0.73 & Livio \& Truran (1994) \\
V2214 Oph & CO & +0.11 & Livio \& Truran (1994) \\
V443 Sct & CO & -1.04 & Livio \& Truran (1994) \\
\hline
\end{tabular}

Notes. $\left.{ }^{(}\right)$The $[\mathrm{Ne} / \mathrm{O}]$ abundances have been computed assuming the solar values in Asplund et al. (2009).

into the circumbinary space. If at any outburst the mass of the ejecta matches or exceeds the accreted mass, then the WD (independent of its composition) cannot increase in mass and reach the Chandrasekhar limit. On the other hand, if the primary star is an $\mathrm{ONeMg} \mathrm{WD}$, it will never explode as a SN Ia even in case of mass gain up to the Chandrasekhar limit of $1.4 M_{\odot}$. Hence, it has become critical to establish the composition of the accreting primary for U Sco because despite its WD mass, high accretion rate and small ejecta mass (a few $10^{-7} M_{\odot}$, Iijima 1999; Anupama \& Dewangan 2000, but see also Diaz et al. 2010), it might not explode as a SN Ia but undergo core collapse at the very most.

Livio \& Truran (1994) cautioned observers about identifying $\mathrm{ONeMg}$ WD progenitors in $\mathrm{CN}$ ejecta that show $\mathrm{Ne}$ emission lines in their optical spectra. They showed that moderate $\mathrm{Ne}$ abundances (with respect to solar) can be explained either by abundance uncertainties or dredged-up material from the underlying CO WD, or with the breakout of the CNO cycle under special conditions. The authors identified a group of "true $\mathrm{ONeMg}$ WDs" in those novae that showed extreme enrichment of $\mathrm{Ne}$ and heavier elements. Table 2 lists the novae used by Livio \& Truran (1994) for their analysis, as well as a number of CNe whose WD and abundances have been determined via photo-ionization modeling of UV and optical observations, simultaneously, by Schwarz and collaborators. The table reports the $[\mathrm{Ne} / \mathrm{O}]$ abundances for each nova as well as the U Sco April 11 relative abundance derived in this paper. From Table 2 it is evident that the $\mathrm{CNe}$ hosting a CO WD are characterized by [Ne/O] abundances $\leq 0$, while those possessing an $\mathrm{ONeMg}$ WD have $[\mathrm{Ne} / \mathrm{O}]>0$. This is also shown in Fig. 3, which plots the distribution of $\mathrm{CNe}$ as a function of their $[\mathrm{Ne} / \mathrm{O}]$ abundance. The shaded histogram of Fig. 3 corresponds to the "fiducial sample" of ONeMg CN white dwarfs; while the black areas correspond to two $\mathrm{CNe}$ that are considered as dubious by Livio \& Truran (1994) on the basis of the high measured $\mathrm{Ne}$ abundances but relatively low values of the total heavy elements enrichment. It should be noted that there were initially three dubious cases identified by Livio \& Truran (1994); but IUE observations of Nova LMC 1990 N.1 
A\&A 532, L11 (2011)

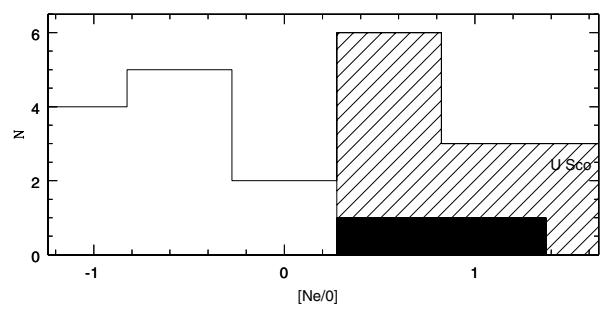

Fig. 3. The histogram of the $\mathrm{CNe}$ distribution as a function of $[\mathrm{Ne} / \mathrm{O}]$ abundance. The white area represents the $\mathrm{CO}$ novae, the shaded are represents the $\mathrm{ONeMg}$ novae, while the black area represents the two $\mathrm{CNe}$ which show extreme $\mathrm{Ne}$ enrichment but relatively small values for the total heavy elements enrichment. See text for more details.

(Starrfield et al. 1992; Vanlandingham et al. 1999) confirmed it to be an $\mathrm{ONeMg}$ nova similar to $\mathrm{V} 463 \mathrm{CrA}$.

The value of $[\mathrm{Ne} / \mathrm{O}]>1$ determined in this paper for U Sco places the binary among the "true Ne novae", hosting an ONeMg WD. It should also be noted that U Sco was observed by IUE during the 1979 outburst and on that occasion Williams et al. (1981) reported absorption lines and P-Cyg profiles from Civ, Sirv and $\mathrm{Nv}$ in their early epoch data. All ONeMg novae - contrary to the CO novae- show a P-Cyg profile phase in their UV spectra after the iron curtain phase and before the transition to the nebular spectrum (Shore 2006). The UV lines displayed at this stage are Sirv 1400, CIv 155 0, AlIII 1860, and MgII 2800 and show a saturated absorption trough with very high terminal velocity (Shore 2006). The IUE spectra of U Sco taken +4 and +6 days after the maximum clearly show P-Cyg profiles with broad absorption troughs that are very similar to the IUE spectra of the ONeMg novae discussed by Shore (2006). Hence, USco should be regarded as a recurrent nova hosting a massive ONeMg WD $\left(M_{\mathrm{WD}} \sim 1.37-1.55 M_{\odot}\right.$, Hachisu et al. 2000; Thoroughgood et al. 2001). The WD will undergo core collapse and will not explode as a SN Ia, unless the current models about accreting ONeMg WDs are significantly in error and the USco mass accretion rate and WD mass prove to be substantially smaller.

It is interesting to note that theoretical studies (e.g. Hachisu et al. 2000; Livio 2000; Thoroughgood et al. 2002, see also Justham \& Podsiadlowski 2008; Walder et al. 2010) have always looked at U Sco and all RNe as likely progenitors of type Ia supernovae. However, observational works on this class of objects seem to show the opposite, i.e. that recurrent novae are not viable SN Ia progenitors. On one hand, Della Valle \& Livio (1996) have shown that the frequency of RNe in the Milky Way, M 31 and the LMC is significantly smaller (by $\sim 1-2$ orders of magnitude) than the supernova Ia rate deduced for these same galaxies. On the other hand, Selvelli et al. (2008) have provided observational evidence that T Pyx ejects more material than it accretes and therefore it cannot explode as a SN Ia. This paper concludes that U Sco, hosting a massive ONeMg WD, cannot explode as a SN Ia either. Therefore, among RNe, only symbiotic recurrent novae "survive" as the possible progenitors of SN Ia (Justham \& Podsiadlowski 2008; Di Stefano 2010), explaining at least some of the observed SN Ia (Patat et al. 2011; Di Stefano 2010). Still, the ultimate fate of RS Oph, the prototype object of the symbiotic RNe, remains uncertain (e.g. Osborne et al. 2006; Justham \& Podsiadlowski 2008).

While the question of how many different stellar systems produce type Ia supernovae remains unsolved, it has been proven critical to establish not only the mass of the WD and the ejecta, but also the primary star composition in candidate SN Ia progenitors. In the case of $\mathrm{RNe}$, in particular, it will be important to establish whether an ONeMg WD is peculiar to U Sco or rather common to the RNe of the same type or to all recurrent novae, as suggested by Webbink (1990).

Acknowledgements. I thank Dr. Bradley Schaefer for having communicated $\mathrm{U}$ Sco broad band magnitudes at the time of X-Shooter observations. I also thank Dr. Massimo Della Valle, Dr. Paola Amico and professor Robert E. Williams for kindly revising the paper before submission; and the anonymous referee for the helpful comments and suggestions. Finally, I thank the ESO Director General, Prof. Tim de Zeew, for approving the observations under DDT 284.D-5041, which allowed the gathering of the unique X-Shooter spectra.

\section{References}

Anupama, G. C., \& Dewangan, G. C. 2000, AJ, 119, 1359

Asplund, M., Grevesse N., Sauval A. J., \& Scott, P. 2009, ARA\&A, 47, 481

Bristow, P., Vernet, J., Moehler, S., et al. 2010, SPIE, 7737E, 34

Della Valle, M., \& Livio, M. 1996, ApJ, 473, 240

Della Valle, M., Pasquini, L., Daou, D., \& Williams, R. E. 2002, A\&A, 390, 155

Diaz, M. P., Williams, R. E., Luna, G. J., Morales, M., \& Takeda, L. 2010, AJ, 140,1860

Di Stefano, R. 2010, ApJ, 719, 474

D’Odorico, S., Dekker H., Mazzoleni, R., et al. 2006, SPIE, 6269E, 98

Dopita, M. A., \& Sutherland, R. S. 2001, Diffuse Matter in the Universe, ed. W. Beiglbock

Hachisu, I., \& Kato, M. 2002, AIP Conf. Proc., 637, 284

Hachisu, I., Kato, M., Kato, T., \& Matsumoto, K. 2000, ApJ, 528, L97

Helton, L., Woodward, C. E., Walter, F. M., et al. 2010, AJ, 140, 1347

Justham, S., \& Podsiadlowski, P. 2008, ASP Conf. Ser., 401, 161

Kingdon, J., \& Williams, R. E. 1997, AJ, 113, 2193

Livio, M., \& Truran, J. W. 1994, ApJ, 425, 797

Livio, M. 2000, in Type Ia Supernovae, Theory and Cosmology (Cambridge University Press), 33

Matsumoto, K., Kato, T., \& Hachisu, I. 2003, PASJ, 55, 297

Modigliani, A., Goldoni, P., Royer, F., et al. 2010, SPIE, 7737E, 56

Munari, U., \& Zwitter, T. 1997, A\&A, 318, 269

Munari, U., Dallaporta, S., \& Castellani, F. 2010, IBVS, 5930

Nomoto, K., \& Kondo, Y. 1991, ApJ, 367, L19

Nomoto, K., Umeda, H., Kobayashi, C., et al. 2000, AIPC, 522, 35

Osborne, J. P., Page, K. L., et al. 2006, ApJ, 727, 124

Osterbrock, D. E., \& Ferland, G. J. 2006, Astrophysics of gaseous nebulae and active galactic nuclei, 2nd edn. (University Science Books)

Patat, F., Chugai, N. N., Podsiadlowski, Ph.., et al. 2011, A\&A, 530, A63

Podsiadlowski, P. 2008, ASP Conf. Ser., 401, 63

Prialnik, D., \& Livio, M. 1995, PASP, 107, 1201

Schaefer, B. E., Pagnotta, A., Xiao, L., et al. 2010, ApJ, 140, 925

Schwarz, G. J. 2002, ApJ, 577, 940

Schwarz, G. J., Shore, S. N., Starrfield, S., et al. 2001, MNRAS, 320, 103

Schwarz, G. J., Shore, S. N., Starrfield, S., \& Vanlandingham, K. M. 2007a, ApJ, 657,453

Schwarz, G. J., Woodward, C. E., Bode, M. F., et al. 2007b, ApJ, 134, 516

Selvelli, P., Cassatella, A., Gilmozzi, R., \& Gonzalez-Riestra, R. 2008, A\&A, 492, 787

Shore, S. N., Bond, H. E., Schwarz, G., et al. 2003, AJ, 125, 1507

Shore, S. N. 2008, in The Classical Novae, ed. M. F. Bode, \& A. Evands, 2nd edn. (Cambridge Univ. Press), 203

Starrfield, S., Politano, M., Truran, J. W., \& Sparks, W. M. 1992, in NASA Goddard Space Flight Center, The Compton Obs. Sci. Workshop, 377

Thoroughgood, T. D., Dhillon, V. S., Littlefair, S. P., Marsh, T. R., \& Smith, D. A. 2001, MNRAS, 327, 1323

Thoroughgood, T. D., Dhillon, V. S., Littlefair, S. P., Marsh, T. R., \& Smith, D. A. 2002, ASP Conf. Proc., 261, 77

Truran, J. W., Livio, M., Hayes, J., Starrfield, S., \& Sparks, W. M. 1988, ApJ, 324,345

Vanlandingham, K. M., Starrfield, S., Shore, S. N., \& Sonneborn, G. 1999, MNRAS, 308, 577

Vanlandingham, K. M., Schwarz, G. J., Shore, S. N., Starrfield, S., \& Wagner, R. M. 2005, ApJ, 624, 914

Vernet, J., Kerber, F., Saitta, F., et al. 2008, SPIE, 7016E, 46

Warner, B. 1995, Cataclysmic Variables Stars (Cambridge: Univ. Press)

Walder, R., Folini, D., Favre, J. M., \& Shore, S. N. 2010, ASPC, 429, 173

Webbink, R. F. 1990, in Physiscs of classical novae, IAU Coll. N. 122, LNP, 369, 405

Yamanaka, M., Uemura, M., Kawabata, K. S., et al. 2010, PASJ, 62, L37

Yaron, O., Prialnik, D., Shara, M. M., \& Kovetz, A. 2005, ApJ, 623, 398 